\title{
DIVERSIDAD GENÉTICA EN MAÍCES NATIVOS MEXICANOS TROPICALES
}

\author{
GENETIC DIVERSITY IN TROPICAL MEXICAN LANDRACES OF MAIZE
}

\author{
Mónica E. González Castro ${ }^{1,2}$, Natalia Palacios Rojas ${ }^{2}$, Armando Espinoza Banda ${ }^{1}$ \\ y Claudia A. Bedoya Salazar ${ }^{2 \star}$
}

\begin{abstract}
${ }^{1}$ Unidad Laguna, Universidad Autónoma Agraria Antonio Narro. Periférico Raúl López Sánchez s/n, Apartado Postal 940. 27054, Torreón, Coahuila. Tel. y Fax: 01 (871) 7297610 Ext. 7676. ${ }^{2}$ Programa Global de Maíz, Centro Internacional de Mejoramiento de Maíz y Trigo. Km 45 carretera México-Veracruz. El Batán. 56130, Texcoco, Edo. de México. Tel. +52 (595) 9521900 Ext. 1112.
\end{abstract}

*Autor para correspondencia (cbedoya@cgiar.org)

\section{RESUMEN}

México es considerado como el centro de origen y domesticación del maíz (Zea mays L.) y uno de los centros más reconocidos de su diversidad. La evaluación de la diversidad en maíces nativos es importante para el planteamiento de estrategias de conservación, caracterización y uso del germoplasma en el mejoramiento genético, dado su potencial como fuente de características nuevas, exóticas y favorables. En este estudio se utilizaron $\mathbf{3 0}$ marcadores moleculares tipo microsatélite con el objetivo de caracterizar la diversidad genética interpoblacional e intrarracial presente en 196 poblaciones del trópico de México representativas de 20 razas de maíz y provenientes de 21 estados de la República Mexicana. Los resultados obtenidos indican que dichas accesiones pueden ser agrupadas en tres áreas ecológicas: Maíces del Golfo de México, Pacífico Sur y Península de Yucatán (A), Maíces de la Zona Noroeste y Occidente (B), y Maíces de Tierras Bajas e Intermedias de Oaxaca y Chiapas (C). A través de las 196 poblaciones se encontró un promedio de 9 alelos por locus y una diversidad genética promedio de 0.57. Se encontró mayor variabilidad entre razas (23.18) que dentro de cada raza ( 0.99 a 8.72). En razas como Jala, Zapalote Grande y Zapalote Chico, se evidenció la erosión genética debido a su limitada distribución geográfica, lo que plantea la necesidad de dedicar esfuerzos a su conservación. Índices de diversidad genética de 0.53 encontrados para los Tuxpeños corroboran que pese a que ha sido ampliamente utilizado en los programas de mejoramiento, aún hay amplio potencial genético por explorar en esta raza.

Palabras clave: Zea mays, diversidad genética, maíces nativos mexicanos.

\section{SUMMARY}

Mexico is considered the center of origin and domestication of maize (Zea mays $\mathrm{L}$.), and it is recognized as one of the most important centers of diversity. Evaluation of native maize diversity is specially important for conservation strategies design, germplasm characterization and its use in breeding; native maize is potentially a source of new, favorable and exotic features. In this study, 30 microsatellite molecular markers were tested to characterize intraracial and between population genetic diversity present in 196 tropical populations representing 20 corn races. Results indicated that these accessions can be grouped into three ecological areas: Gulf of México, South Pacific and Yucatán Peninsula (A), the Northwestern and Western (B), and Intermediate Lowland Oaxaca and Chiapas (C). Average number of alleles per locus within the populations was 9 . Average genetic diversity of 20 tropical Mexican maize races was 0.57 across all accessions. There was greater variability among races (23.18) than within each race (0.99 to 8.72). Genetic erosion due to limited geographical distribution for Zapalote and Jala races was evident, thus indicating the need for preservation efforts. Genetic diversity indices of $\mathbf{0 . 5 3}$ found for Tuxpeño germplasm confirm that although they have been widely used in breeding programs, there is untapped diversity in this race.

Index words: Zea mays, genetic diversity, Mexican landraces.

\section{INTRODUCCIÓN}

El maíz (Zea mays L.) es uno de los tres cereales más importantes del mundo, que se produce en casi 100 millones de hectáreas en 125 países. En México es de gran importancia por su historia, tradición e impacto social y económico; donde se siembran 7.2 millones de hectáreas, con una producción de 23.3 millones de toneladas (FAOSTAT, 2010). En la región tropical húmeda del sureste del país se siembran anualmente 2.5 millones de hectáreas.

México es considerado como el centro de origen y domesticación, y uno de los centros más importantes de diversidad del maíz (Matsuoka et al., 2002). La variabilidad genética de maíz constituye una riqueza para la población mundial, y puede ser la base para lograr la soberanía alimentaria de México, en especial ante los cambios climáticos (Preciado y Montes, 2011). La evaluación de dicha diversidad es importante para los programas de mejoramiento genético, por su potencial como fuente de características nuevas, exóticas y favorables (Vigouroux et al., 2008).

La revalorización de los recursos genéticos se ha visto beneficiada por los avances en técnicas biotecnológicas. El uso de marcadores moleculares ha permitido caracterizar la diversidad en materiales mejorados, complejos genéticos, variedades de polinización abierta y materiales nativos (Matsuoka et al., 2002; Reif et al., 2006; Vigoroux et al., 2008; Sharma et al., 2010; Wen et al., 2012). Dentro de dichos marcadores, los SSRs (microsatélites o secuencias repetidas cortas, por sus siglas en inglés) se han utilizado extensamente para la caracterización de diversidad genética y 
en la descripción de estructura genética de poblaciones, por tener alta confiabilidad, reproducibilidad y automatización (Bedoya et al., 2010). Los estudios con SSRs pueden presentar algunas diferencias cuando se comparan entre ellos, especialmente en el número de alelos reportados y en la heterocigocidad esperada, debido a la naturaleza (dinucléotida, triculcléotida, etc.) de los SSRs seleccionados (Vigoroux et al., 2002; Reif et al., 2006), así como al tamaño de muestra, las accesiones seleccionadas y los individuos seleccionados para representar cada accesión (Reif et al., 2006).

La variabilidad de maíz en México ha sido caracterizada en estudios morfológicos, bioquímicos, y moleculares. Herrera et al. (2000) identificaron 11 caracteres morfológicos que recomiendan usar para la evaluación de la diversidad genética; Doebley et al. (1984) analizaron el patrón de 13 isoenzimas en 94 accesiones (34 razas) de maíz mexicano, y reportaron una gran variación tanto dentro de razas (72\%) como entre razas (27\%). Sánchez et al. (2000) estudiaron morfológicamente y por isoenzimas a 209 accesiones de 59 razas, y encontraron mayor diversidad entre poblaciones que entre razas. Reif et al. (2006) utilizó 25 marcadores SSR para caracterizar 25 accesiones de 24 razas mexicanas, y también reportaron gran variabilidad en el material utilizado.

El objetivo de este estudio fue caracterizar la diversidad genética interpoblacional e intrarracial presente en 196 accesiones de maíz tropical provenientes de 21 estados mexicanos, que representan 20 razas, mediante el uso de 30 SSRs, para ampliar el conocimiento sobre la diversidad genética de los maíces tropicales de México y contribuir al planteamiento de estrategias de conservación, caracterización y uso de las mismas en el mejoramiento genético.

\section{MATERIALES Y MÉTODOS}

Se analizaron 20 razas de maíz de México de las 25 razas descritas por Wellhausen et al. (1951), de un total de 196 poblaciones tropicales (Cuadro 1). En este estudio el término "población" hace referencia a las variedades nativas que son mantenidas por los agricultores, y cada una de estas poblaciones se registra como una accesión en los bancos de germoplasma. El material fue proporcionado por el banco de germoplasma del Centro Internacional de Mejoramiento de Maíz y Trigo (CIMMYT), y sembrado en la Estación Experimental Agua Fría-CIMMYT de clima tropical húmedo, ubicada en el municipio de Venustiano Carranza, Puebla (entre $20^{\circ} 23^{\prime} 12^{\prime \prime}$ y $20^{\circ} 37^{\prime} 36^{\prime \prime} \mathrm{LN}$ y entre $97^{\circ} 31^{\prime} 54^{\prime \prime}$ y $\left.97^{\circ} 48^{\prime} 42^{\prime \prime} \mathrm{LO}\right)$, a $110 \mathrm{msnm}$.

Se usaron 15 plantas elegidas al azar de cada accesión para hacer una muestra compuesta de ADN ("bulk"). Se cosecharon hojas frescas y sanas de plantas de alrededor de $30 \mathrm{~d}$ de edad. El tejido vegetal se congeló a $-80{ }^{\circ} \mathrm{C}$, luego se liofilizó y se molió finamente para la extracción de $\mathrm{ADN}$ bajo el método de CTAB (CIMMYT, 2006). El ADN se cuantificó con el espectrofotómetro NanoDrop ${ }^{\circledR}$ (Thermo Scientific, Wilmington, DE) y se realizó la reacción en cadena de la polimerasa (PCR, por sus siglas en inglés) de acuerdo con el protocolo estandarizado por CIMMYT cuando se utiliza un secuenciador automático de $\mathrm{ADN}$ para el análisis de fragmentos (CIMMYT, 2006). Se utilizaron 30 marcadores SSR (Cuadro 2) de la base de datos genéticos y genómicos para maíz dsiponible en línea (Maize Genetics and Genomics Database; http://www.maizegdb.org/).

Las PCRs se hicieron en un termociclador MJ Research ${ }^{\circledR}$ (Ramsey, Minnesota, USA), según se describe en CIMMYT (2006). Los productos de la PCR se separaron por electroforesis capilar con un secuenciador automático ABI3100 Genetic Analyzer ${ }^{\circledR}$ (Foster City, California, USA) equipado con el programa GeneMapper ${ }^{\circledR}$, programa que toma como referencia un estándar interno de tamaño (ROX $500^{\mathrm{m}}$ ) para calcular automáticamente el tamaño en pares de bases y altura de pico de los fragmentos encontrados.

Las frecuencias alélicas de cada fragmento presente en cada muestra conjunta de ADN se calcularon con el programa Freqs-R (Franco et al., 2005); este programa se basa en que la altura de cada pico es proporcional a la frecuencia del alelo en la muestra conjunta de ADN. Cada frecuencia alélica fue posteriormente transformada en 15 individuos dialélicos con el programa FtoL-R (Franco et al., 2007), de tal forma que el conjunto de los 15 individuos cumplieron las frecuencias alélicas dentro de cada "bulk" y la heterocigosidad esperada bajo las condiciones de equilibrio de Hardy-Weinberg (Franco et al., 2007). Esta transformación es necesaria ya que diferentes programas para el análisis de diversidad no aceptan frecuencias alélicas de muestras conjuntas de ADN, porque pueden presentar múltiples alelos por locus.

Los parámetros de diversidad genética, como número de alelos, diversidad genética (heterocigosidad esperada), así como el análisis de varianza molecular (AMOVA) y las distancias genéticas entre accesiones/poblaciones obtenidas mediante el uso de la proporción de alelos compartidos, se calcularon con el paquete estadístico de libre acceso PowerMarker Versión 3.25 (Liu y Muse, 2005). El programa DARwin 5.0 (http://darwin.cirad.fr/Home.php) se utilizó para generar el dendograma por el método Neighbor-Joining basado en la matriz de distancias genéticas, que fue hecho con el programa PowerMarker con el propósito de obtener una estructura de las relaciones entre las 20 razas mexicanas de maíces tropicales. 
Cuadro 1. Razas de maíz procedencia por Estado.

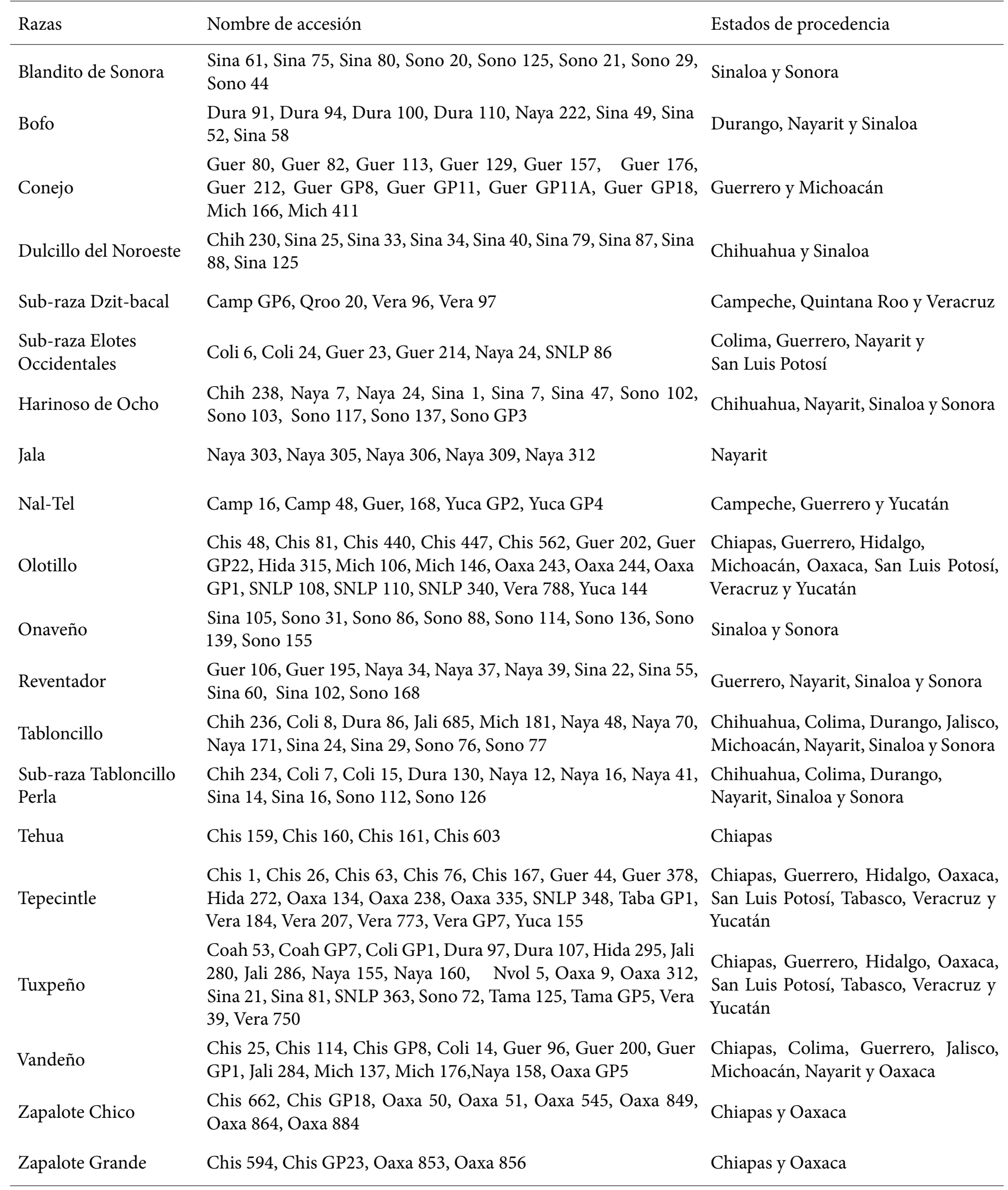


Cuadro 2. SSRs utilizados, posición del marcador en cada cromosoma (Bin), unidad de repetición, fluoróforo y rango de tamaño en pares de bases, utilizado como referencia en el análisis de los datos.

\begin{tabular}{|c|c|c|c|c|}
\hline Marcador & Bin & Unidades de repetición & Fluoróforos & Tamaño en pares de bases \\
\hline nc133 & 2.05 & GTGTC & Hex & $99-120$ \\
\hline phi008 & 5.03 & GGC & Fam & $100-104$ \\
\hline phi029 & 3.04 & AG/AGCG & Ned & $150-161$ \\
\hline phi034 & 7 & CCT & Ned & $125-143$ \\
\hline phi056 & 1.01 & CCG & Hex & $241-258$ \\
\hline phi063 & 10.02 & TATC & Fam & $154-180$ \\
\hline phi065 & 9.03 & CACTT & Fam & $128-148$ \\
\hline phi075 & 6 & $\mathrm{CT}$ & Fam & 203-235 \\
\hline phi076 & 4.11 & GAGCGG & Hex & $156-177$ \\
\hline phi079 & 4.05 & CATCT & Ned & $177-195$ \\
\hline phi084 & 10.04 & GAA & Hex & $148-157$ \\
\hline phi090 & 2.08 & ATATC & Hex & $125-140$ \\
\hline phil14 & 7.02 & GCCT & Hex & $129-171$ \\
\hline phi127 & 2.08 & AGAC & Ned & $110-127$ \\
\hline phi102228 & $3.04-3.05$ & AAGC & Hex & $122 / 131$ \\
\hline phi108411 & 9.06 & AGCT & Fam & $116 / 123$ \\
\hline phi109188 & 5 & AAAG & Hex & $145-181$ \\
\hline phi299852 & 6.08 & AGC & Ned & $102-147$ \\
\hline phi374118 & 3.03 & ACC & Ned & $214-238$ \\
\hline umc1161 & 8.06 & GCTGGG & Ned & $128-149$ \\
\hline umc1196 & 10.07 & CACACG & Fam & $137-173$ \\
\hline umc1266 & 3.06 & CAG & Fam & $120-147$ \\
\hline umc1304 & 8.02 & TCGA & Ned & $121-141$ \\
\hline umc1332 & 5.04 & CTA & Hex & $126-147$ \\
\hline umc1367 & 10.03 & CGA & Fam & $143-158$ \\
\hline umc1447 & 5.03 & CTT & Fam & $115-119$ \\
\hline umc1545 & 7 & AAGA & Hex & $64-80$ \\
\hline umc1917 & 1.04 & CTG & Ned & $119-143$ \\
\hline umc2047 & 1.09 & GACT & Hex & $119-133$ \\
\hline umc2250 & 2.04 & ACG & Fam & $130-150$ \\
\hline
\end{tabular}

\section{RESULTADOS Y DISCUSIÓN}

\section{Análisis de agrupamiento}

Los resultados obtenidos en este estudio indican que con base en los marcadores SSR y el uso de muestras conjuntas de ADN, las poblaciones de maíces tropicales mexicanos pueden ser agrupadas en tres áreas ecológicas: Golfo de México, Pacífico Sur y Península de Yucatán (A); Zona Noroeste y Occidente (B); y Tierras Bajas e Intermedias de Oaxaca y Chiapas (C) (Figura 1).
Esta distribución corresponde en su gran mayoría a las distribuciones previamente reportadas para estas razas (Ortega et al., 1991). En el área ecológica A se identificaron las razas (número de accesiones): Jala (8), Nal-Tel (9), Olotillo (10), Tepecintle (16), Tuxpeño (17), Vandeño (18) y la sub-raza Dzit-Bacal (5). Dentro de este componente se identificaron también algunas razas distribuidas en la parte del Pacífico Sur: Olotillo (10), Tepecintle (16), Tuxpeño (17) y Vandeño (18). El grupo B comprende a las razas: Blandito de Sonora (1), Bofo (2), Dulcillo del Noroeste (4), Harinoso de Ocho (7), Onaveño (11), Reventador (12), Tabloncillo (13) y Tabloncillo Perla (14). 
a)

A

Maíces del Golfo de México

Pacífico Sur y Península de Yucatán

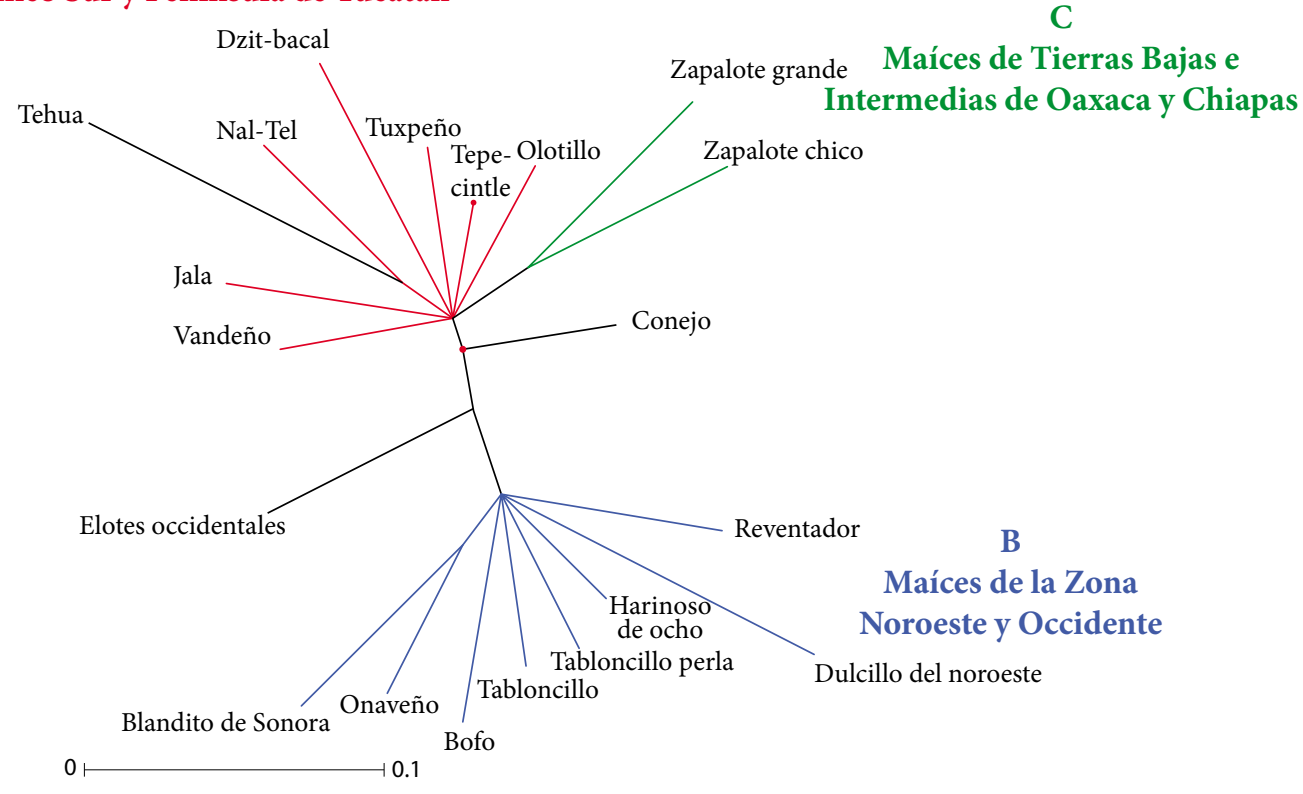

b)

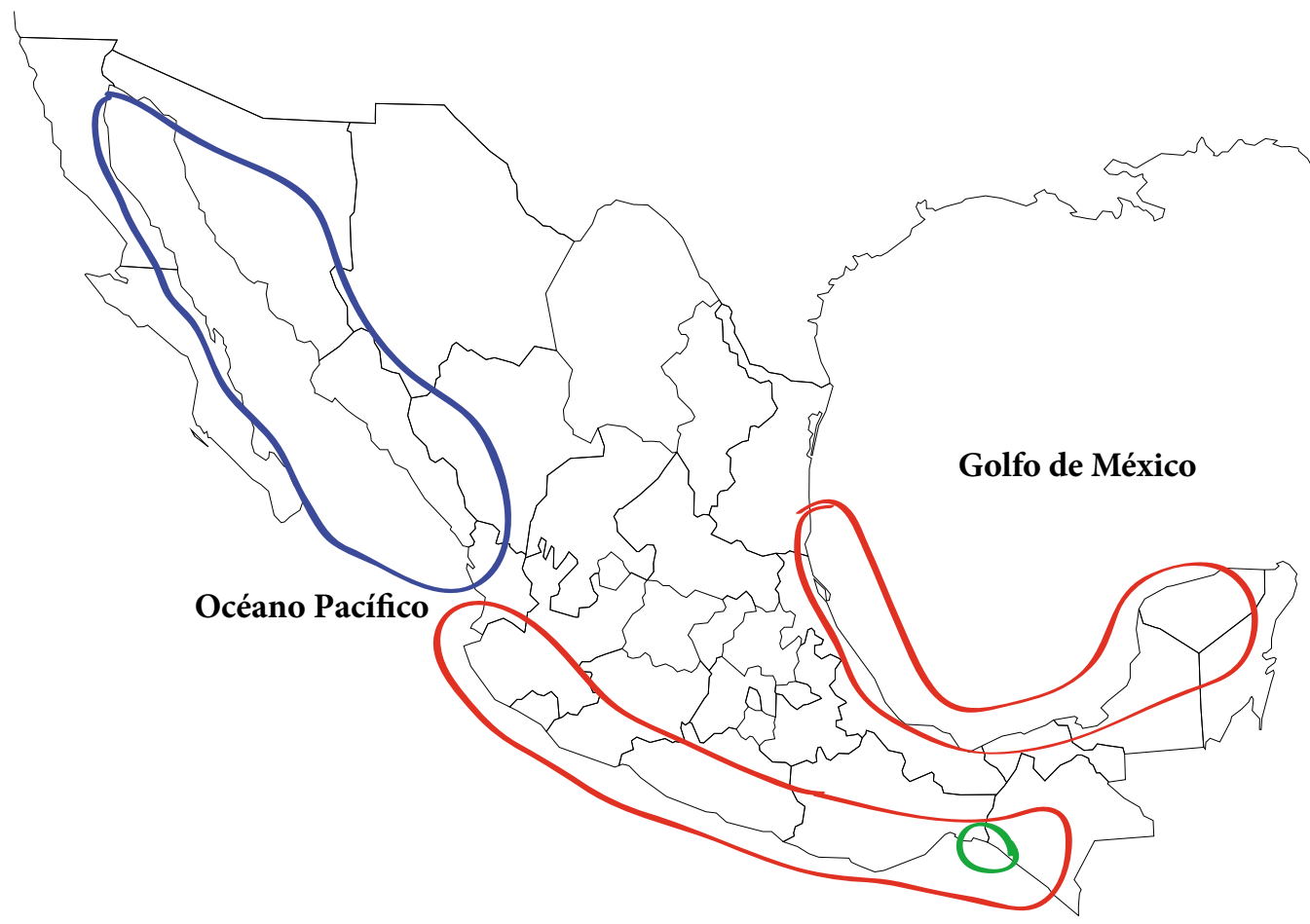

Figura 1. a) Análisis de agrupamiento por razas de 196 poblaciones de maíz tropical mexicano. b) Distribución de los grupos identificados en el mapa de México. Grupo A “Maíces del Golfo de México, Pacífico Sur y Península de Yucatán” (rojo), grupo B "Maíces de la Zona Noroeste y Occidente” (azul), y grupo C “Maíces de Tierras Bajas e Intermedias de Oaxaca y Chiapas" (verde). 
El grupo C está conformado por las razas: Zapalote Chico (19) y Zapalote Grande (20), las cuales pertenecen a razas mestizas prehistóricas descritas por Wellhausen et al. (1951). Tanto Zapalote Chico como Zapalote Grande se encuentran en las tierras bajas de las costas de Oaxaca y Chiapas (Perales et al., 2003).

Las razas Conejo (3), Tehua (15) y Elotes Occidentales (6) no agruparon en ninguna de las tres áreas ecológicas anteriormente descritas. Conejo es una de las razas clasificadas por Wellhausen et al. (1951) en el grupo denominado razas no bien definidas, cuya ubicación geográfica es en tierras poco fértiles de las costas y tierra caliente de Michoacán a Oaxaca (Ortega et al., 1991). La raza Tehua se ha encontrado en el Estado de Chiapas, en altitudes de 600 a $100 \mathrm{~m}$ (Wellhausen et al., 1951), y corresponde a una raza antigua y casi extinta (CONABIO, 2008) que presenta características especiales reflejadas en su composición alélica, que ha sido mantenida por los pequeños agricultores pese al marcado desplazamiento de variedades nativas por la presencia de híbridos en sitios con alta productividad (Bellon y Hellin, 2011).

La sub-raza de Harinoso de Ocho, Elotes Occidentales, tiene su centro de distribución en la altiplanicie de Jalisco a elevaciones de 1200 a 1600 msnm, y también se ha encontrado en El Bajío (Ortega et al., 1991). Sin embargo, dentro de las accesiones de la raza Elotes Occidentales utilizadas en este estudio había colectas del Estado de Guerrero, en altitudes entre 29 y $267 \mathrm{~m}$. El aumento del área de distribución y rango de altitud donde habitualmente se ha cultivado esta raza podría haber originado una mayor diversificación, que en este caso podría explicar que la raza no agrupe dentro de los componentes de maíces del Noroeste de México.

\section{Caracterización de la diversidad genética}

Para las 196 accesiones de maíz tropical mexicano el promedio del número de alelos por locus fue de 9; para los tres grupos según zonas ecológicas $(\mathrm{A}, \mathrm{B}, \mathrm{C})$ el número de alelos por locus varió de 5.63 para el grupo C a 8.20 para el grupo A (Cuadro 3). Estos valores difieren de otros estudios anteriores, como el de Reif et al. (2006) quienes reportaron 7.84 alelos por locus al usar 25 SSRs y estudiar solamente 25 accesiones de maíces de México de diferentes adaptaciones (valles altos, trópico y sub-trópico). Sharma et al. (2010) reportaron el mayor número de alelos por locus (13), hasta donde se tiene conocimiento, al estudiar 48 accesiones de la región de la India del Estado de Sikkim, con 42 SSRs. En estudios realizados de germoplasma en la ex-Yugoslavia se han reportado valores de solo 2.5 alelos por locus al utilizar 25 SSRs en solo 21 poblaciones (Ignjatović-Micić et al., 2008).
Del total de alelos detectados en este estudio, alrededor de $50 \%$ presentaron frecuencias menores de $5 \%$, a los que se les denominó alelos raros o de baja frecuencia (Cuadro 3). Alelos raros en accesiones mexicanas fueron también reportados por Santacruz-Varela et al. (2004), quien encontró 5 de 58 alelos en maíces palomeros. Sánchez et al. (2000) reportaron que de 303 alelos, 194 fueron raros. El grupo A presentó el mayor número de alelos únicos, lo que refleja el potencial de estas razas para ser incorporadas en programas de mejoramiento, ya que los alelos raros pueden otorgar una ventaja adaptativa en condiciones adversas o diferentes.

La diversidad genética promedio evaluada en las tres áreas ecológicas definidas presentó un mayor valor en el grupo $\mathrm{B}$, que contiene el mayor número de razas $(0.58 \pm$ $0.01)$; por su parte, el grupo $C$ presentó el valor más bajo (0.52 \pm 0.02$)$ (Cuadro 3$)$. La diversidad genética promedio de las 20 razas de maíz tropical mexicano fue de 0.57 a través de todas las accesiones. Los mayores índices de diversidad encontrados fueron en las razas: Onaveño $(0.58 \pm$ $0.03)$, Harinoso de Ocho (0.57 \pm 0.04$)$, Reventador $(0.57 \pm$ $0.05)$, Tabloncillo $(0.57 \pm 0.04)$, Blandito de Sonora $(0.56$ $\pm 0.04)$, Bofo $(0.56 \pm 0.05)$ y Dulcillo del Noroeste $(0.56 \pm$ $0.05)$; el menor índice fue para Tehua $(0.46 \pm 0.05)$. Reif et al. (2006) reportaron 0.61 de diversidad genética con 24 razas mexicanas de maíz. Aunque la diversidad genética encontrada en este estudio presentó valores intermedios a los antes reportados en maíces mexicanos, la comparación entre estudios siempre debe hacerse con precaución debido a las diferencias del material objeto de investigación, y al tamaño de muestra, número y naturaleza de los marcadores utilizados.

Los índices de diversidad dentro de cada raza en el estudio de Reif et al. (2006) y los del presente estudio difieren notoriamente. Jala (0.52), Olotón (0.53) y Zapalote Chico (0.53) fueron las razas con mayor índice de diversidad según Reif et al. (2006), mientras que en nuestro estudio Jala presentó un índice de diversidad más bajo (0.48) con respecto a otras razas, y Zapalote Chico dio un índice de 0.51. En el estudio de Reif et al. (2006), la comparación de diversidad genética se hizo entre razas provenientes de un rango mayor de adaptabilidad (valles altos, alturas intermedias y trópico), lo que favorece la diferenciación genética entre ellas, y ello se refleja en mayores índices de diversidad dentro de cada raza. En cambio, en este estudio las comparaciones realizadas corresponden a razas de maíz de adaptación restringida (trópico y de alturas bajas-intermedias). Cabe mencionar que la raza Jala es la que presentó la menor diversidad genética en este estudio, y esto puede ser consecuencia del hecho de que su adaptación es solamente para una región muy específica, el Valle del Jala, Nayarit (Ortega, 2003). 
Cuadro 3. Áreas ecológicas, razas relacionadas, alelos encotrados, alelos únicos e índices de diversidad genética de 20 razas de maíz tropical mexicano.

\begin{tabular}{|c|c|c|c|c|}
\hline Áreas ecológicas & Razas & $\begin{array}{l}\text { Alelos encontrados } \\
\text { por locus }\end{array}$ & Alelos únicos ${ }^{\dagger}$ & $\begin{array}{c}\text { Diversidad genética } \pm \\
\text { desviación estándar }\end{array}$ \\
\hline \multirow{8}{*}{$\begin{array}{l}\text { A) Maíces del Golfo de } \\
\text { México, Pacífico Sur y } \\
\text { Península de Yucatán }\end{array}$} & Dzit-Bacal & 4.13 & & $0.50 \pm 0.04$ \\
\hline & Jala & 4.50 & & $0.48 \pm 0.05$ \\
\hline & Nal-Tel & 4.17 & & $0.47 \pm 0.04$ \\
\hline & Olotillo & 6.70 & & $0.54 \pm 0.05$ \\
\hline & Tepecintle & 6.27 & & $0.53 \pm 0.05$ \\
\hline & Tuxpeño & 6.67 & & $0.53 \pm 0.04$ \\
\hline & Vandeño & 5.87 & & $0.50 \pm 0.05$ \\
\hline & Total & 8.20 & 20 & $0.53 \pm 0.03$ \\
\hline \multirow{9}{*}{$\begin{array}{l}\text { B) Maíces de la Zona } \\
\text { Noroeste y Occidente }\end{array}$} & Blandito de Sonora & 5.40 & & $0.56 \pm 0.04$ \\
\hline & Bofo & 5.50 & & $0.56 \pm 0.05$ \\
\hline & Dulcillo del Noroeste & 5.33 & & $0.56 \pm 0.05$ \\
\hline & Harinoso de Ocho & 5.63 & & $0.57 \pm 0.04$ \\
\hline & Onaveño & 5.60 & & $0.58 \pm 0.03$ \\
\hline & Reventador & 5.57 & & $0.57 \pm 0.05$ \\
\hline & Tabloncillo & 5.77 & & $0.57 \pm 0.04$ \\
\hline & Tabloncillo Perla & 5.53 & & $0.56 \pm 0.06$ \\
\hline & Total & 7.73 & 7 & $0.58 \pm 0.01$ \\
\hline \multirow{3}{*}{$\begin{array}{l}\text { C) Maíces de Tierras } \\
\text { Bajas e Intermedias de } \\
\text { Oaxaca y Chiapas }\end{array}$} & Zapalote Chico & 5.07 & & $0.51 \pm 0.04$ \\
\hline & Zapalote Grande & 4.43 & & $0.51 \pm 0.04$ \\
\hline & Total & 5.63 & 4 & $0.52 \pm 0.002$ \\
\hline \multirow[t]{5}{*}{ (SN) Maíces de México } & Conejo & 5.87 & & $0.54 \pm 0.04$ \\
\hline & Elotes Occidentales & 4.87 & & $0.54 \pm 0.05$ \\
\hline & Tehua & 3.77 & & $0.46 \pm 0.05$ \\
\hline & Total & 6.53 & 1 & $0.54 \pm 0.05$ \\
\hline & Promedio & 9 & & $0.57 \pm 0.17$ \\
\hline
\end{tabular}

${ }^{\dagger}$ Número de alelos únicos encontrados en zonas geográficas y razas seleccionadas. $\mathrm{SN}$ = Razas no agrupadas.

El grupo A presentó el mayor número de alelos por locus (8.20), lo que indica que hay mayor variabilidad alélica en esta agrupación debido a la amplia distribución geográfica de las razas que componen este grupo. Contrariamente, el grupo $\mathrm{C}$ mostró el menor número de alelos por locus, lo que puede deberse a que estas razas pertenecen a una microrregión, el Istmo de Tehuantepec (tierras bajas costeras de Oaxaca y Chiapas) (López et al., 2005). Esto es consistente con la premisa de que a mayor rango de dispersión geográfica de una especie vegetal, ocurre una mayor variabilidad (Franco e Hidalgo, 2003).

Si bien los índices de diversidad dan una idea de la variabilidad presente en un conjunto de germoplasma, también es conveniente conocer como está distribuida o estructura- da mediante el AMOVA, que dado el número de individuos por accesión (15), el número de accesiones por raza (12 en promedio) y el número de razas utilizadas en este estudio, dicho análisis es bastante robusto para representar la variabilidad genética dentro y entre razas.

De esta manera se encontró mayor variabilidad entre razas (23.18) que dentro de cada raza (máximo 8.72 y mínimo 0.99) (Cuadro 4). El área ecológica B presentó el mayor porcentaje de variación (38.23\%), lo que sugiere una gran variabilidad genética que se puede explorar ampliamente. El menor porcentaje se encontró dentro del área ecológica C, con $3.88 \%$. Esto confirma resultados anteriores, en el sentido de que a mayor número de razas y más amplia su distribución geográfica, mayor es la variación dentro del 
grupo. Así se ratifica que la limitada distribución geográfica de los Zapalotes haya conducido a un porcentaje de variación muy bajo. Por otro lado, las razas no agrupadas indican una diversidad genética que no está presente en los tres grupos definidos previamente, según sus áreas ecológicas.

De acuerdo con la varibilidad intrarracial detectada, se puede afirmar que las razas Jala, Zapalote Grande y Nal-Tel están perdiendo variabilidad genética, al poseer los porcentajes de variación más bajos $(0.99,1.06$ y 1.17). La raza Jala se caracteriza por el gran tamaño de su mazorca, que llega a medir hasta $60 \mathrm{~cm}$ de longitud, y por plantas de gran altura que alcanzan hasta de $5 \mathrm{~m}$ (Rice et al., 2006). Debido a la erosión genética que ha sufrido esta raza a través del tiempo, se ha visto una marcada reducción en la longitud

Cuadro 4. Análisis de varianza molecular (AMOVA) de 20 razas de maíz tropical mexicano y de áreas ecológicas según su ubicación geográfica.

\begin{tabular}{lc}
\hline Fuentes de variación & Variación (\%) \\
\hline Entre razas & 23.18 \\
Entre Áreas Ecológicas & 12.10 \\
Jala & 0.99 \\
Nal-Tel & 1.17 \\
Olotillo & 7.22 \\
Dzit-Bacal & 1.27 \\
Tepecintle & 8.72 \\
Tuxpeño & 7.25 \\
Vandeño & 4.57 \\
Dentro Área Ecológica (A) & 34.52 \\
Blandito de Sonora & 4.14 \\
Bofo & 3.25 \\
Dulcillo del Noroeste & 4.57 \\
Harinoso de Ocho & 4.71 \\
Onaveño & 3.03 \\
Reventador & 5.09 \\
Tabloncillo & 4.45 \\
Tabloncillo Perla & 3.46 \\
Dentro Área Ecológica (B) & 38.26 \\
Zapalote Chico & 2.36 \\
Zapalote Grande & 1.06 \\
Dentro Área Ecológica (C) & 3.88 \\
Conejo & 5.60 \\
Elotes Occidentales & 1.96 \\
Tehua & 1.95 \\
Dentro de razas no agrupadas (SN) & 11.27 \\
\hline
\end{tabular}

de la mazorca y poca adaptabilidad a otras regiones. Su cultivo hoy en día es microrregional, limitado al Valle del Jala, Nayarit, con una superficie de siembra de aproximadamente 30 ha por año (Aguilar-Castillo et al., 2006).

Las razas Jala y Nal-Tel también forman parte de los maíces de usos especiales; por ejemplo, Jala se utiliza para elotes, pozole y gorditas de horno, y la raza Nal-Tel para elotes y elaboración de atole (Ortega, 2003). Los maíces para usos especiales pueden tener problemas para su conservación, ya que los agricultores normalmente los siembran para autoconsumo, en muy pequeñas superficies cercanas a sus maíces principales destinados a la comercialización, por lo que la posibilidad de contaminación es alta.

Las razas Tuxpeño, Tepecintle y Olotillo presentaron mayor diversidad intrarracial, con porcentajes de variación de $7.25,8.72$ y 7.22 , respectivamente (Cuadro 4). La raza Tuxpeño se caracteriza por su grano dentado (blanco o amarillo) y mazorcas de tipo cilíndrico, además destaca por ser el maíz nativo mexicano de más amplia adaptabilidad pues se encuentra en 19 tipos climáticos diferentes (Wen et al., 2012). La productividad, tipo de planta, la tolerancia a la sequía y enfermedades del Tuxpeño han sido destacados como atributos positivos en programas de mejoramiento. Pese a que se ha utilizado ampliamente, estudios genéticos recientes han demostrado que al menos en uno de los programas de mejoramiento de maíz de mayor impacto mundial, como lo es el de CIMMYT, existe gran diversidad genética de esta raza que aún no se ha explotado (Wen et al., 2012). Este estudio comprueba la diversidad genética de Tuxpeño (0.53) (Cuadros 3 y 4) y ratifica lo reportado por Wen et al. (2012) en el sentido de que no sólo dentro de los Tuxpeños mexicanos sino también en introducciones latinoamericanas (Brasil, Ecuador, Guatemala y Venezuela), existe un gran potencial para ser usados en los programas de mejoramiento.

Los resultados obtenidos sugieren acciones importantes dentro de programas de conservación y aprovechamiento dentro de los programas de mejoramiento del maíz. Sin embargo, para mantener la diversidad es necesario diseñar mecanismos de intervención que no solamente estimulen la conservación del material genético, sino que también contribuyan a mejorar los resultados productivos y económicos para los agricultores (Aguirre et al., 2010), al permitir el acceso a la diversidad de las variedades nativas de la región, capacitación en técnicas de selección y manejo de semilla para mantener las caracterítiscas valiosas (Bellon et al., 2004).

\section{CONCLUSIONES}

Las razas tropicales analizadas mediante marcadores SSR, definieron tres grupos que coinciden con su distribución 
geográfica en la República Mexicana, y comprueba que las diferencias ambientales contribuyen en gran proporción al patrón general de la diversidad del maíz. Así mismo, se determinó que en las razas estudiadas existe mayor diversidad genética interracial que intrarracial.

Los resultados evidenciaron la erosión genética en razas como Jala y Tehua, y la necesidad de dedicar esfuerzos a su conservación. Así mismo es importante considerar entre las diferentes estrategias de conservación y uso, el complejo genético Zapalote que puede llegar a estar en riesgo por su limitada distribución geográfica.

Para un mejor aprovechamiento de la diversidad genética existente, una caracterización genotípica debe de ir ligada a una evaluación fenotípica dirigida a caracteres de interés, lo que permitiría identificar el germoplasma nativo con gran potencial por poseer alelos favorables para condiciones adversas. La diversidad genética del germoplasma de maíz $y$ en especial la diversidad encontrada en este trabajo, justifican el emprender estudios más amplios, asociados con características de rusticidad de las plantas, tolerancia a enfermedades, alto rendimiento, y calidad industrial y nutricional, para asegurar la conservación de los maices nativos a través de su uso.

\section{AGRADECIMIENTOS}

Al Dr. Suketoshi Taba y el MSc. Víctor Chávez, por sus aportes en la selección y clasificación de las razas utilizadas en este estudio. Al personal técnico de la Estación Experimental de Agua Fría. Este trabajo se hizo con el apoyo de AgroBio-México. MEGC fue financiada por beca de maestría del CONACYT.

\section{BIBLIOGRAFÍA}

Aguilar-Castillo J A, A Carballo-Carballo, F Castillo-González, A Santacruz- Varela, J A Mejía-Contreras, J Crossa-Hiriartte, G Baca-Castillo (2006) Diversidad fenotípica y variantes distintivas de la raza Jala de maíz. Agric. Téc. Méx. 32:57-66.

Aguirre-Moreno V C, F Rincón-Sánchez, R Ramírez-Segoviano, O G Colón-Alvarado, M G Razo-Marín (2010) Modelo para la Conservación de Maíces Criollos en el Sureste de Coahuila. Ed. UAAAN-COLPOS-SINAREFI. $49 \mathrm{p}$.

Bedoya S C, C Mir, A Charcosset, M Warburton (2010) Migración del Maíz a partir de su Centro de Origen, Evidencias Históricas, Genéticas y Paleobotánicas. El Cultivo del Maíz, Temas Selectos, Volumen 2. Ed. Mundi Prensa. 227 p.

Bellon-Mauricio R, J A Aguirre, M Smale, J Berthaud, M Rosas, J Mendoza, A Solano, R Martínez (2004) Intervenciones participativas para la conservación de maíz en fincas en los Valles Centrales de Oaxaca, México. In: Manejo de la Diversidad de los Cultivos en los Agroecosistemas Tradicionales. J L Chávez Servia, J Tuxill, D I Jarvis (eds). Instituto Internacional de Recursos Fitogenéticos. Cali, Colombia. 118 p.

Bellon M, J Hellin (2011) Planting hybrids, keeping landraces: Agricultural modernization and tradition among small-scale maize farmers in Chiapas, Mexico. World Develop. 39:1434-1443

CIMMYT, Centro Intrenacional de Mejoramiento de Maíz y Trigo
(2006) Protocolos de Laboratorio. Laboratorio de Genética Molecular Aplicada del CIMMYT. 3a ed. El Batán, Texcoco, México. $92 \mathrm{p}$.

CONABIO, Comision Nacional de Biodiversidad (2008) Proyecto FZ002 "Conocimiento de la Diversidad y distribución del maíz nativo y sus parientes silvestres en México". Responsable: Dr. A Ortega Corona (INIFAP). Comisión Nacional para la Conservación y el Uso de la Biodiversidad. México, D. F. 81 p.

Doebley J F, M M Goodman, C W Stuber (1984) Isoenzymatic variation in Zea (Gramineae). Syst. Bot. 9:203-218.

FAOSTAT, División de Estadísticas de la FAO (Organización de las Naciones Unidas para la Alimentación y la Agricultura) (2010) Los datos finales correspondientes al año 2010. Disponible en: http://faostat.fao.org/DesktopDefault aspx?PageID=567\&lang=es\#ancor. (Mayo del 2012).

Franco T L, R Hidalgo (2003) Análisis Estadístico de Datos de Caracterización Morfológica de Recursos Fitogenéticos. Boletín técnico no. 8. Instituto Internacional de Recursos Fitogenéticos. Cali, Colombia. $89 \mathrm{p}$.

Franco J, M Warburton, P Dubreuil, S Dreisigacker (2005) User's Manual for the FREQS-R Program for Estimating Allele Frequencies for Fingerprinting and Genetic Diversity Studies Using Bulked Heterogeneous Populations. CIMMYT, México, D. F.

Franco J, M Warburton, S Dreisigacker (2007) User's Manual for the FtoL-R Program for Generating a "dummy" Data Set Consisting of Allele Lengths for Hypothetical Individuals. CIMMYT, Mexico, D. F. 15 p.

Herrera C B E, F Castillo, J Sánchez, R Ortega, M M Goodman (2000) Caracteres morfológicos para valorar la diversidad entre poblaciones de maíz en una región: caso de la raza Chalqueño. Rev. Fitotec. Mex. 23:335-354.

Ignjatović-Micić D, S Mladenović, A Nikolić, V Lazić-Jančić (2008) SSR analysis for genetic structure and diversity determination of maize local populations from former Yugoslavia territories. Russian J. Genet. 11:1317-1324.

Liu K, S Muse (2005) PowerMarker: an integrated analysis environment for genetic marker analysis. Bioinformatics 21:2128-2129.

López R G, A Santacruz, A Muñoz, F Castillo, L Córdova, H Vaquera (2005) Caracterización morfológica de poblaciones nativas de maíz del Istmo de Tehuantepec, Oaxaca. Interciencia 30:284290.

Maize Genetics and Genomics Database. Disponible en: http://www. maizegdb.org/ (Mayo del 2012)

Matsuoka Y, Y Vigouroux, M M Goodman, J Sánchez, E Buckler, J Doebley (2002) A single domestication for maize shown by multilocus microsatellite genotyping. Proc. Nat. Acad. Sci. USA 99:6080-6084.

Ortega P R (2003) Diversidad de maíz en México: Causas, estado actual y perspectivas. In: Sin Maíz no hay País. Culturas Populares, CONACULTA, México, D. F. pp:123-154.

Ortega P R, J J Sánchez, F Castillo, J M Hernández (1991) Estado actual de los estudios sobre maíces nativos de México. In: R Ortega P, G Palomino, F Castillo, V A González, M Livera (eds). Avances en el Estudio de los Recursos Fitogenéticos de México. Sociedad Mexicana de Fitogenética, A C. Chapingo, México. pp:161 185.

Perales H R, S B Brush, C O Qualset (2003) Landraces of maize in central Mexico: An altitudinal transect. Econ. Bot. 57:7-20.

Preciado O R E, S Montes (2011) Reseña del libro "Amplitud, Mejoramiento, Usos y Riesgos de la Diversidad Genética de Maíz en México". Rev. Fitotec. Mex. 34, Núm. 4. 2 p.

Reif J C, M L Warburton, X C Xia, D A Hoisington, J Crossa, S Taba, J Muminović, M Bohn, M Frisch, A E Melchinger (2006) Grouping of accessions of Mexican races of maize revisited with SSR markers. Theor. Appl. Genet. 113:177-85.

Rice E B, M E Smith, S E Mitchell, S Kresovich (2006) Conservation and change: a comparison of in situ and ex situ conservation of Jala maize germplasm. Crop Sci. 46:428-436.

Sánchez G J J, M M Goodman, C W Stuber (2000) Isozymatic and morphological diversity in the races of maize of Mexico. Econ. Bot. 54:43-59.

Santacruz-Varela A, M P Widrlechner, R J Salvador, K E Ziegler, M J Millard, P K Bretting (2004) Phylogenetic relationships 
among North American popcorns and their evolutionary links to Mexican and South American popcorns. Crop Sci. 44:14561467.

Sharma L, B M Prasanna B Ramesh (2010) Analysis if phenotypic and microsatellite-based diversity of maize landraces in India, especially from the North East Himalaya region. Genetica 138:619-331.

Vigouroux Y, J C Glaubitz, Y Matsuoka, M M Goodman, J Sánchez, J Doebley (2008) Population structure and genetic diversity of new world maize races assessed by DNA microsatellites. Amer. J. Bot. 95:1240-1253.

Vigouroux Y, M McMullen, C T Hittinger, K Houchins, L Schulz, S Kresovich, Y Matsuoka, J Doebley (2002) Identifying genes of agronomic importance in maize by screening microsatellites for evidence of selection during domestication. Proc. Nat. Acad. Sci. USA 99:9650-9655.

Wellhausen E J, L M Roberts, E Hernández, en colaboración con P C Mangelsdorf (1951) Razas de Maíz en México. Su Origen. Características y Distribución. Folleto Técnico No.5. Secretaría de Agricultura y Ganadería. Oficina de Estudios Especiales. México, D. F. 237 p.

Wen W, J Franco, V H Chavez-Tovar, J Yan, S Taba (2012) Genetic characterization of a core set of a tropical maize race tuxpeño for further use in maize improvement. PLoS ONE 7(3): e32626. doi:10.1371/journal.phone.0032626. 